\title{
LRRK2 but not ATC16L1 is associated with Paneth cell defect in Japanese Crohn's disease patients
}

\author{
Ta-Chiang Liu, ${ }^{1}$ Takeo Naito, ${ }^{2}$ Zhenqiu Liu, ${ }^{3}$ Kelli L. VanDussen, ${ }^{1}$ Talin Haritunians, ${ }^{3}$ Dalin Li, ${ }^{3}$ \\ Katsuya Endo, ${ }^{2}$ Yosuke Kawai, ${ }^{4}$ Masao Nagasaki, ${ }^{4}$ Yoshitaka Kinouchi, ${ }^{5}$ Dermot P.B. McGovern, ${ }^{3}$ \\ Tooru Shimosegawa, ${ }^{2}$ Yoichi Kakuta, ${ }^{2}$ and Thaddeus S. Stappenbeck ${ }^{1}$ \\ 'Department of Pathology and Immunology, Washington University School of Medicine, St. Louis, Missouri, USA. 'Division \\ of Gastroenterology, Tohoku University Graduate School of Medicine, Sendai, Japan. ${ }^{3}$. Widjaja Family Foundation \\ Inflammatory Bowel and Immunobiology Research Institute, Cedars-Sinai Medical Center, Los Angeles, California, USA. \\ ${ }^{4}$ Department of Integrative Genomics, Tohoku Medical Megabank Organization, Tohoku University, Sendai, Japan. \\ ${ }^{5}$ Health Administration Center, Center for the Advancement of Higher Education, Tohoku University, Sendai, Japan.
}

BACKGROUND. Morphological patterns of Paneth cells are a prognostic biomarker in Western Crohn's disease (CD) patients, and are associated with autophagy-associated ATC16L1 and NOD2 variants. We hypothesized that genetic determinants of Paneth cell phenotype in other ethnic CD cohorts are distinct but also involved in autophagy.

METHODS. We performed a hypothesis-driven analysis of 56 single nucleotide polymorphisms (SNPs) associated with CD susceptibility or known to affect Paneth cell function in 110 Japanese CD patients who underwent ileal resection. We subsequently performed a genome-wide association analysis. Paneth cell phenotype was determined by defensin- 5 immunofluorescence. Selected genotype-Paneth cell defect correlations were compared to a Western CD cohort $(n=164)$.

RESULTS. The average percentage of abnormal Paneth cells in Japanese CD was similar to Western CD $(P=0.87)$, and abnormal Paneth cell phenotype was also associated with early recurrence ( $P$ $=0.013)$. In contrast to Western CD, ATC16L1 T300A was not associated with Paneth cell defect in Japanese CD $(P=0.20)$. Among the 56 selected SNPs, only LRRK2 M2397T showed significant association with Paneth cell defect $\left(P=3.62 \times 10^{-4}\right)$, whereas in the Western CD cohort it was not $(P=0.76)$. Pathway analysis of $L R R K 2$ and other candidate genes with $P$ less than $5 \times 10^{-4}$ showed connections with known CD susceptibility genes and links to autophagy and TNF- $\alpha$ networks.

CONCLUSIONS. We found dichotomous effects of ATC16L1 and LRRK2 on Paneth cell defect between Japanese and Western CD. Genes affecting Paneth cell phenotype in Japanese CD were also associated with autophagy. Paneth cell phenotype also predicted prognosis in Japanese CD.

sponsor has no role in study design collection, analysis, and interpretation of data, the writing of the manuscript, and the decision to submit the manuscript for publication.

Authorship note: T.C. Liu and T. Naito contributed equally to this work. Y. Katuka and T.S. Stappenbeck contributed equally to this work.

Conflict of interest: M. Nagasaki received research grants from Toshiba Corporation during the conduct of the study.

Submitted: November 23, 2016

Accepted: February 2, 2017

Published: March 23, 2017

\section{Reference information:}

FUNDING. Helmsley Charitable Trust, Doris Duke Foundation (grant 2014103), Japan Society for the Promotion of Science (KAKENHI grants JP15H04805 and JP15K15284), Crohn's and Colitis Foundation grant 274415, NIH (grants 1R56DK095820, K01DK109081, and UL1 TR000448).

\section{Introduction}

Crohn's disease (CD) and ulcerative colitis are 2 main classical types of inflammatory bowel disease (IBD) $(1,2)$. The etiology of IBD involves genetic susceptibility and environmental triggers. Over 200 single nucleotide polymorphisms (SNPs) have been associated with susceptibility to IBD (3-7). This complex genetic network indicates that IBD likely encompasses more than the 2 classical subtypes. Therefore, novel, rationally designed biomarkers that can lead to disease stratification and personalized treatments are needed (8). One candidate method to subtype $\mathrm{CD}$ is to define the morphological patterns of small intestinal Paneth cells based on the intracellular distribution of granules containing antimicrobial proteins (Paneth cell phenotypes) (7). Paneth cells are specialized secretory cells located at the bases of the crypts of Lieberkühn in the small intestine (9-11). These cells produce a wide repertoire of antimicrobial 


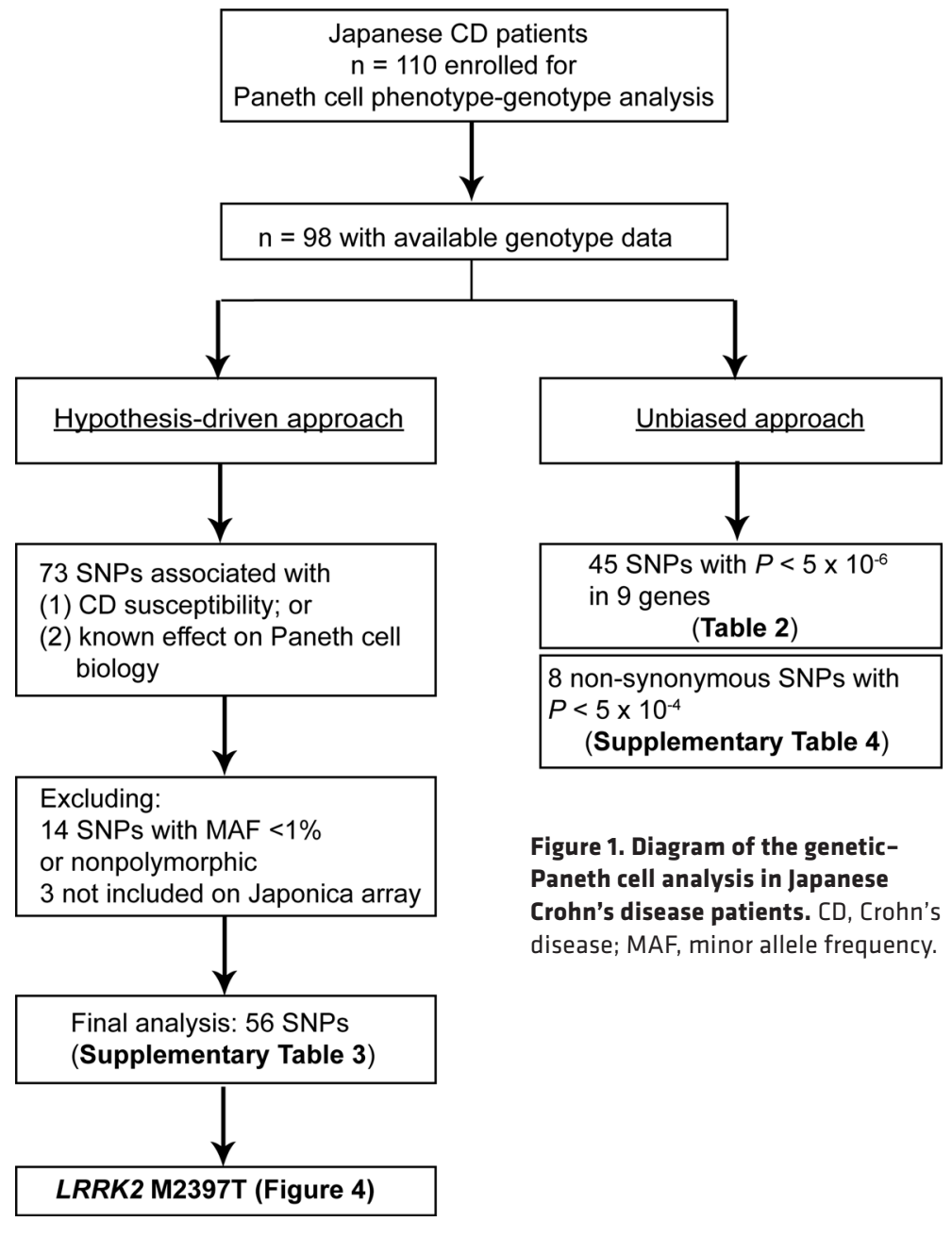

peptides, such as lysozyme and $\alpha$-defensins, to modulate the intestinal microbiome (12-15) and thus are important mediators of the host innate immune response $(16,17)$.

We have previously shown that in European ancestry CD patients from North America, abnormal Paneth cell phenotype is associated with variants in ATG16L1 and NOD2 genes, both of which are CD susceptibility genes involved in autophagy $(18,19)$. Furthermore, we have also demonstrated that abnormal Paneth cell phenotype is associated with mucosal dysbiosis and aggressive disease course $(19,20)$. Thus, Paneth cell phenotype is a biologically and clinically relevant biomarker that can stratify CD patients.

To broaden the applications of Paneth cell phenotype in $\mathrm{CD}$, a more detailed understanding of the genetic determinants of Paneth cell phenotype and further clinical validation are critical. However, as over $90 \%$ of Western $\mathrm{CD}$ cases are composed of patients of European ancestry who harbor ATG16L1 T300A and/or NOD2 CD-risk variants $(3,4)$, identification of novel genetic determinants in this population is challenging. Thus, studying $\mathrm{CD}$ patients from other ethnicities, especially those who possess a spectrum of susceptibility genes distinct from those found in European ancestry CD, would be helpful in identifying novel genetic determinants of Paneth cell phenotype. The genetic landscape of $C D$ patients from Korea and Japan is partially overlapping but largely different than that of European ancestry $C D$ (5, 21-23). In particular, the NOD2 variants associated with European ancestry $\mathrm{CD}$ are not polymorphic in Japanese populations (24). In addition, while ATG16L1 $\mathrm{T} 300 \mathrm{~A}$ is polymorphic in Japanese cohorts, it has not been associated with CD susceptibility (25). In contrast, SNPs that are more frequently associated with CD in Asian populations include those that tag TNFSF15, IL23R, ATG16L2, STAT3, GPR35, MHC Class $I I$, and ZNF365, among others $(5,21-23,25)$. Therefore, a Japanese CD patient cohort represents a unique cohort to identify novel genetic determinants of Paneth cell phenotypes.

In this study, we hypothesized that novel genetic determinants of Paneth cell defect exist in Japanese CD, and that abnormal Paneth cell phenotype would be associated with poor outcome in Japanese CD patients. We further hypothesized that these novel genetic determinants of Paneth cell defect in Japanese CD would also be associated with autophagy as in North American CD. We found that the prevalence of abnormal Paneth cell phenotype in Japanese $C D$ was similar to North American CD. Abnormal Paneth cell phenotype also correlated with more aggressive outcome in Japanese CD. Our hypothesis-driven analysis identified that autophagyassociated $L R R K 2$, but not $A T G 16 L 1$, was associated with Paneth cell defect in Japanese CD. In contrast, in North American CD, ATG16L1 but not LRRK2 was associated with Paneth cell defect. In addition, unbiased genome-wide analysis for Paneth cell phenotype genetic associations identified additional candidate SNPs predicted to interact with known CD susceptibility genes and jointly affect autophagy or TNF- $\alpha$ signaling.

\section{Results}

Paneth cell defects were prevalent in Japanese CD patients. The overall work flow for the determination of genetic associations of Paneth cell defect in Japanese CD patients is outlined in Figure 1. We first determined the percentage of normal Paneth cells in resection specimens of adult Japanese CD subjects using a previously reported method of immunofluorescence localization for defensin-5 (20). The staining for defensin-5 was specific for Paneth cells and the results were highly correlative to that obtained by lysozyme immunofluorescence $\left(R^{2}=0.6451 ; P<0.0001\right)$ (Supplemental Figure 1 ; supplemental material available online with this 
A

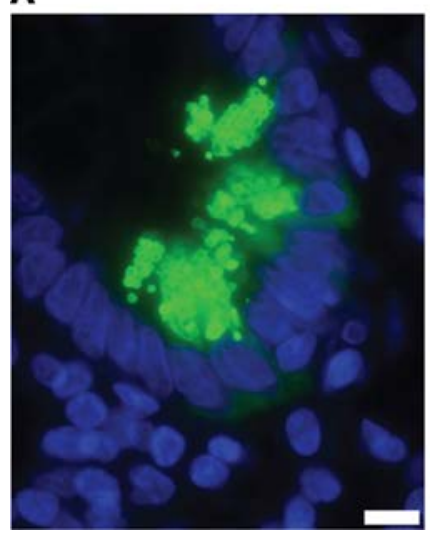

B

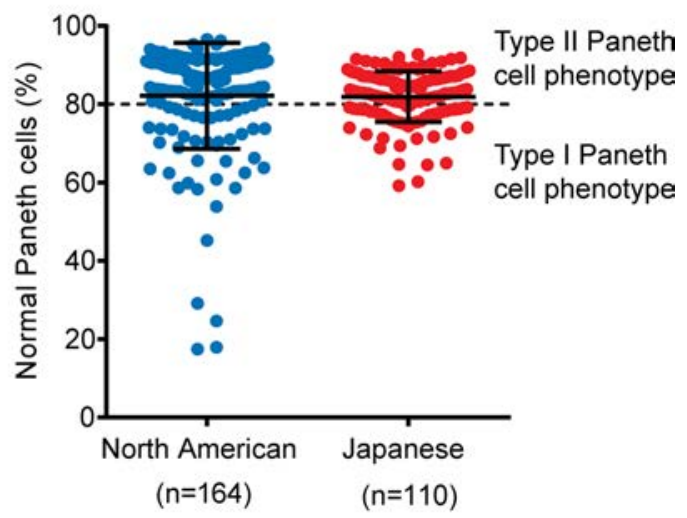

Figure 2. Type I Paneth cell phenotype was prevalent in Japanese Crohn's disease (CD). (A) Representative images of defensin-5 immunofluorescence on Japanese $C D$ resection specimens. Scale bar: $10 \mu \mathrm{m}$. (B) The distribution of percentage of normal Paneth cells in North American $(n=164)$ and Japanese CD $(n=110) . P=0.87$ by unpaired $t$ test. Error bars represent \pm SEM.

article; https://doi.org/10.1172/jci.insight.91917DS1). There was a similar range of intracellular staining patterns to those previously observed in multiple North American CD cohorts $(19,20)$ (Figure 2A). The average percentage of normal Paneth cells was similar between Japanese and North American CD subjects $(82.13 \pm 1.06$ vs. $81.9 \pm 0.55 ; P=0.87$ ) (Figure $2 \mathrm{~B}$ ). As in previous studies, we utilized a cutoff of greater than or equal to $20 \%$ (high abundance) abnormal Paneth cells to define a type I Paneth cell phenotype (type II is $<20 \%$ abnormal Paneth cells). We found that the prevalence of type I Paneth cell phenotype in Japanese CD subjects was similar to a North American cohort (33\% vs. 26\%; $P=0.26$ ) (Figure 2B). We also examined potential correlations between the clinical data obtained for the Japanese CD subjects and Paneth cell phenotypes (Table 1). Of note, there was no significant difference in the demographics and clinical phenotype (Montreal classification) between patients with type I and type II Paneth cell phenotypes at time of resection. In addition, we also examined whether Paneth cell phenotype and/or individual morphology category correlated with the presence or absence of granuloma. We found that while there was a trend in that the percentage of diminished Paneth cell morphology inversely correlated with the presence of granuloma as we have shown previously (19), overall there was no significant correlation between Paneth cell phenotype or each Paneth cell morphology category and granuloma (Supplemental Figure 2).

Type I Paneth cell phenotype was associated with poor clinical outcome in Japanese CD. To determine whether Paneth cell phenotype also correlated with clinical outcome in patients undergoing resections in Japanese $\mathrm{CD}$, we analyzed the clinical outcome only for those patients who had received postoperative prophylactic therapy, as postoperative prophylaxis has been shown to be associated with outcome (26). We listed the characteristics of the patients that were included for outcome analysis in Supplemental Table 1. We found that in Japanese CD patients, type I Paneth cell phenotype was also associated with shorter time to disease recurrence after resection $(P=0.013$; hazard ratio $=2.10,95 \% \mathrm{CI}=1.04-4.24)$ (Figure 3$)$. This association remained significant in multivariate analysis (Supplemental Table 2), thus replicating the finding that we have previously shown in North American CD patients (19).

ATG16L1 T300A was not associated with Paneth cell defect in Japanese CD. We next examined the potential associations of type I Paneth cell phenotype with 56 SNPs, selected based on known CD susceptibility associations $(3,5,21-23,27-29)$ or known association with Paneth cell function (17) (Supplemental Table 3). These SNPs include coding variants for ATG16L1 (T300A) associated with Paneth cell defects in North American CD cohorts and in genetic mouse models $(18,19,30)$. Among the Japanese CD patients with available genotype data $(n=98)$, we found no significant difference between the numbers of the ATG16L1 T300A risk allele and the percentage of normal Paneth cells $\left(R^{2}=0.01717 ; P=0.20\right)$ (Figure 4A). In contrast, in North American CD with wild-type NOD2 (to eliminate potential confounding factors from NOD2; $n=97$ ), the numbers of $A T G 16 L 1$ T300A risk allele correlated with the percentage of normal Paneth cells $\left(R^{2}=0.04387 ; P=\right.$ 0.0395 ; Figure 4B), a finding that we have shown previously with a smaller CD cohort (18). Of note, the allele frequency of $A T G 16 L 1 \mathrm{~T} 300 \mathrm{~A}$ in our Japanese CD cohort was $40 \%$, a level comparable to what has been published (27). Therefore, in contrast to the North American CD, ATG16L1 T300A was not associated with the percentage of normal Paneth cells or type I Paneth cell phenotype in Japanese CD. NOD2, another CD susceptibility gene previously shown to be associated with Paneth cell defect in North American CD patients, is nonpolymorphic in Japanese patients $(31,32)$, and thus was not included on the Japonica SNP array. 
Table 1. Characteristics of all Japanese Crohn's disease patients included in this study

\begin{tabular}{|c|c|c|c|}
\hline & Type I Paneth cell phenotype & Type II Paneth cell phenotype & $P$ value \\
\hline$n$ & 31 & 79 & \\
\hline Sex (male) & $22(71.0 \%)$ & $60(76.0 \%)$ & 0.630 \\
\hline Mean age at onset (range) & $23.8(21.5-26.2)$ & $23.1(21.7-24.6)$ & 0.612 \\
\hline \multicolumn{4}{|l|}{ Disease Onset ${ }^{A}$} \\
\hline A1 & $4(12.9 \%)$ & $11(13.9 \%)$ & 1.000 \\
\hline \multicolumn{4}{|l|}{ Disease Location } \\
\hline L1 & $3(9.7 \%)$ & $12(15.2 \%)$ & 0.550 \\
\hline L2 & 0 & 0 & NA \\
\hline L3 & $28(90.3 \%)$ & $67(84.8 \%)$ & 0.550 \\
\hline \multicolumn{4}{|l|}{ Disease Behavior } \\
\hline Current smoking & $9(36 \%)$ & $16(32 \%)$ & 0.798 \\
\hline \multicolumn{4}{|l|}{ Treatment received prior to surgery } \\
\hline Biologics (Bio) & $12(38.7 \%)$ & $22(27.9 \%)$ & 0.359 \\
\hline Immunomodulator (IM) & $5(16.1 \%)$ & $7(8.9 \%)$ & 0.313 \\
\hline Elemental diet (ED) & $12(38.7 \%)$ & $18(22.8 \%)$ & 0.102 \\
\hline \multicolumn{4}{|l|}{ Treatment combination prior to surgery } \\
\hline Only Bio & $5(16.1 \%)$ & $13(16.5 \%)$ & 1.000 \\
\hline Only IM & 0 & $2(2.5 \%)$ & 1.000 \\
\hline Only ED & $6(19.4 \%)$ & $13(16.5 \%)$ & 0.781 \\
\hline Bio+IM & $1(3.2 \%)$ & $4(5.1 \%)$ & 1.000 \\
\hline Bio+ED & $2(6.5 \%)$ & $4(5.2 \%)$ & 1.000 \\
\hline ED & $13(41.9 \%)$ & 26 (32.9\%) & 0.385 \\
\hline \multicolumn{4}{|l|}{ Treatment combination } \\
\hline Only Bio & $9(29.0 \%)$ & $17(21.5 \%)$ & 0.457 \\
\hline Only IM & $1(3.2 \%)$ & $2(1.8 \%)$ & 1.000 \\
\hline Only ED & $5(16.1 \%)$ & $14(17.7 \%)$ & 1.000 \\
\hline Bio+IM & $1(3.2 \%)$ & $7(8.9 \%)$ & 0.437 \\
\hline Bio+ED & $4(12.9 \%)$ & 9 (11.4\%) & 1.000 \\
\hline$E D+I M$ & $2(6.5 \%)$ & $1(1.3 \%)$ & 0.191 \\
\hline $\mathrm{Bio}+\mathrm{IM}+\mathrm{ED}$ & $2(6.5 \%)$ & $2(2.5 \%)$ & 0.315 \\
\hline
\end{tabular}

${ }^{A}$ Disease onset, location, and behavior are reported according to the Montreal classification.

Western CD susceptibility allele LRRK2 M2397T was associated with Paneth cell defect in Japanese CD. Interestingly, the only SNP within the pool of 56 selected alleles for hypothesis-driven correlation analysis that showed significant association with Paneth cell defects in Japanese CD was LRRK2 M2397T (Figure 4C). The LRRK2 M2397T SNP (rs3761863) is a missense susceptibility allele for European ancestry CD $(3,33)$, and Lrrk2 knockout mice have defective Paneth cells (34). In the Japanese CD cohort, we found that the numbers of the T (risk) allele $L R R K 2 \mathrm{M} 2397 \mathrm{~T}$ correlated with the percentage of normal Paneth cells $\left(R^{2}=\right.$ $0.247 ; P=3.62 \times 10^{-4}$; Figure $\left.4 \mathrm{C}\right)$. The results suggest that 2 defective copies of the $L R R K 2$ gene product may be required to illicit Paneth cell defects in Japanese CD patients. 


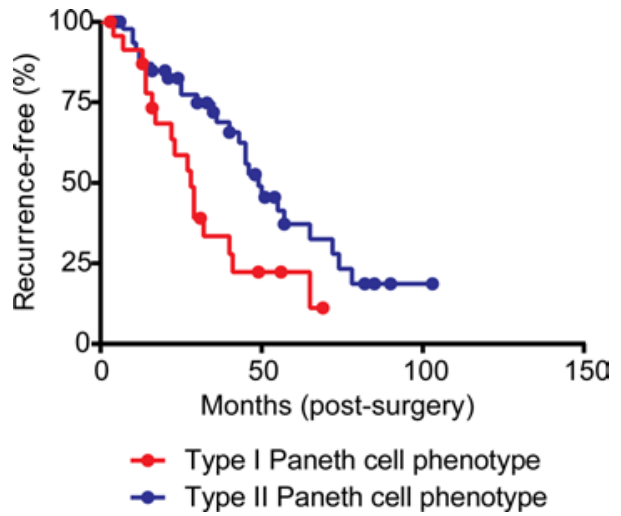

— Type II Paneth cell phenotype
Figure 3. Paneth cell phenotypes correlated with time to disease recurrence after surgery in Japanese Crohn's disease (CD). The Kaplan-Meier curves of time to disease recurrence after resection was determined in the CD cases that received prophylaxis after surgery $(n=76)$. Patients with type I Paneth cell phenotype showed a significantly shorter time to disease recurrence $(P=0.013$ by log-rank test).

To determine whether this correlation was universal, we next analyzed the correlation of LRRK2 M2397T and Paneth cell defects in the North American CD cohort (with wild-type NOD2 as described above). Surprisingly, there was no association between the numbers of LRRK2 M2397T risk allele and the degree of Paneth cell defect $\left(R^{2}=\right.$ 0.02054; $P=0.76$; Figure 4D). As ATG16L1 T300A and NOD2 SNPs are known to be associated with Paneth cell defects in North American CD cohorts, we performed further analyses with the North American CD patients with ATG16L1 T300A and/or NOD2 risk alleles ( $n=116$ with $L R R K 2$ M2397T status available) as well as the patients without any risk alleles for $A T G 16 L 1 \mathrm{~T} 300 \mathrm{~A}$ or NOD2 ( $n=15$ with $L R R K 2 \mathrm{M} 2397 \mathrm{~T}$ status available). There was no association of LRRK2 M2397T and the percentage of normal Paneth cells when all patients (including $A T G 16 L 1$ T300A and/or NOD2 risk alleles) were analyzed ( $P=0.13$; Supplemental Figure 3A) or when only the subset of patients without $A T G 16 L 1$ T300A and NOD2 risk alleles were analyzed $(P=$ 0.82; Supplemental Figure 3B). However, given the relatively small sample size with wild-type $A T G 16 L 1$ T300A and NOD2, further investigation with a greater sample size is needed. Therefore, our data suggest that there are dichotomous effects of the ATG16L1 and LRRK2 susceptibility alleles on Paneth cell defects within Japanese and North American CD cohorts. Interestingly, LRRK2 M2397T itself was not associated with outcome ( $P=0.5931)$ (Supplemental Table 2).

Unbiased genome-wide association identified additional candidate genes associated with Paneth cell defect in Japanese $C D$. We subsequently performed an unbiased GWAS analysis to identify additional potential novel SNPs that could be associated with the degree of Paneth cell defects. Although no SNPs reached genome-wide significance $\left(<5 \times 10^{-8}\right), 45$ SNPs were identified as candidates $\left(P \leq 5 \times 10^{-6}\right)$, as well as 8 nonsynonymous SNPs

Figure 4. ATC16L1 T300A and LRRK2 M2397T genotypes showed dichotomous effects on Paneth cell defects in Japanese and North American Crohn's disease (CD) patients. (A) The numbers of the ATG16L1 T300A risk allele did not correlate with the percentage of normal Paneth cells $\left(R^{2}=0.01717\right.$ and $P$ $=0.20$ by linear regression) in Japanese CD, while (B) in North American CD without the common CD-associated NOD2 risk allele, the numbers of the ATG16L1 T300A risk allele correlated with the percentage of normal Paneth cells $\left(R^{2}=0.04387\right.$ and $P=0.0395$ by linear regression). In contrast, (C) the numbers of the LRRK2 M2397T risk allele negatively correlated with the percentage of normal Paneth cells in Japanese CD $\left(R^{2}=0.247, P=3.62 \times 10^{-4}\right.$ by linear regression). (D) However, in North American CD, the numbers of the $L R R K 2$ M2397T risk allele did not correlate with the percentage of normal Paneth cells $\left(R^{2}=0.02054, P=\right.$ 0.76 by linear regression). Error bars represent \pm SEM.
A

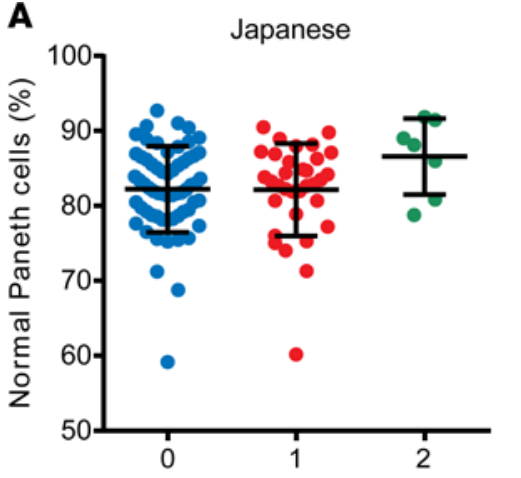

Numbers of ATG16L1 T300A risk allele

C

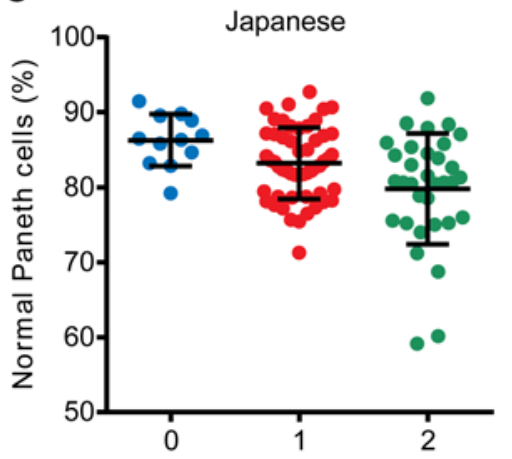

Numbers of LRRK2 M2397T risk allele

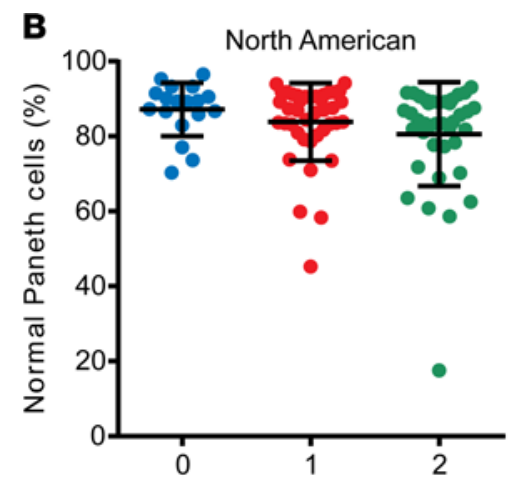

Numbers of ATG16L1 T300A risk allele

\section{D}

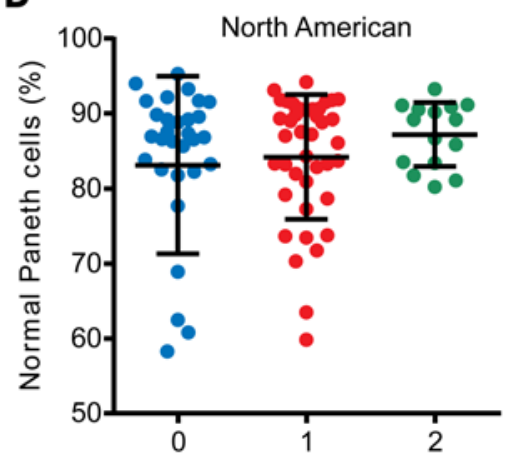

Numbers of $L R R K 2$ M2397T risk allele 
A

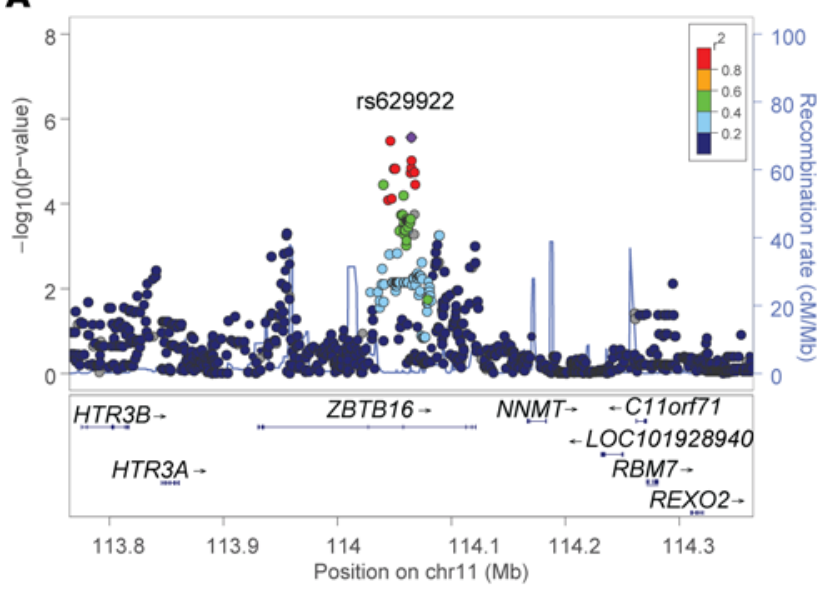

C

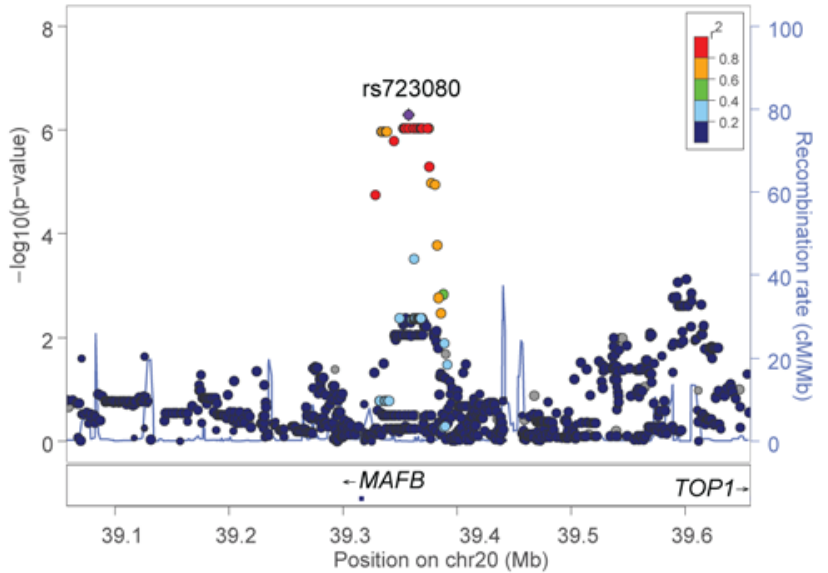

E

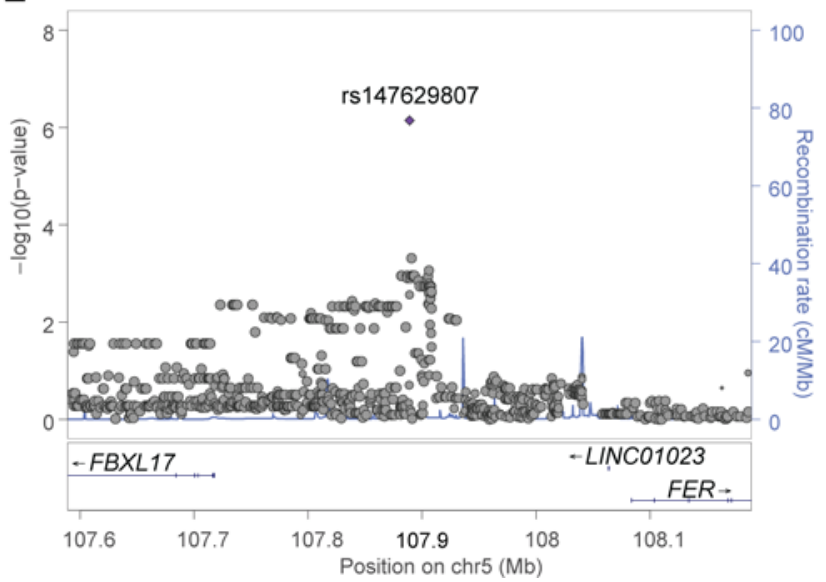

B

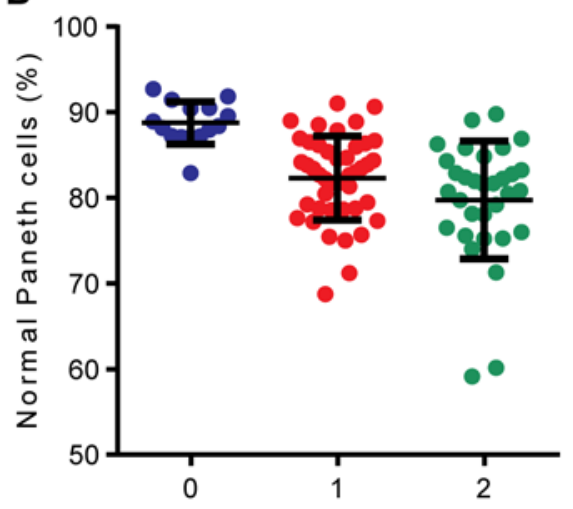

Numbers of ZBTB16 risk allele

D

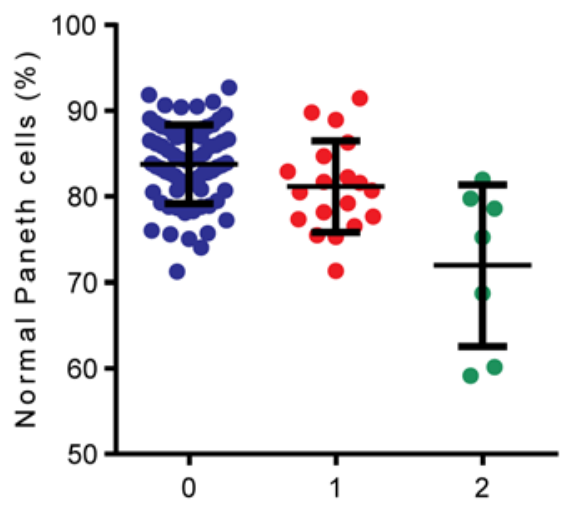

Numbers of $M A F B$ risk allele

$\mathbf{F}$

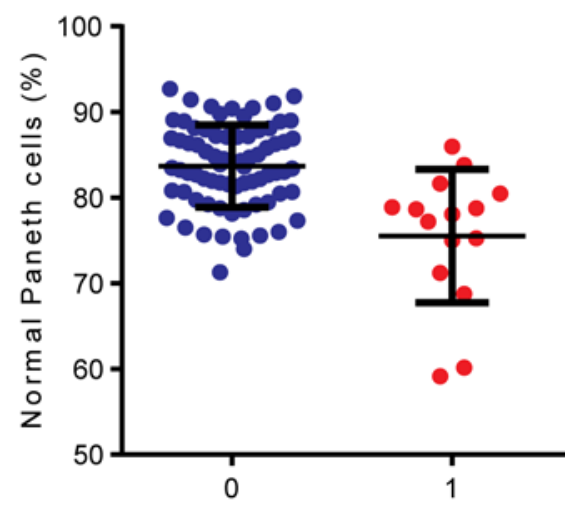

Numbers of FER risk allele

Figure 5. Genome-wide association analysis results for Paneth cell defect in Japanese Crohn's disease (CD) patients. Locus zoom plots of $P$ values around selected top associated SNPs from Paneth cell phenotype regression analysis. The top associated SNPs for (A) ZBTB16, (C) MAFB, and (E) FER are shown as purple diamonds and the remaining SNPs are shown as circles, with color indicating the level of linkage disequilibrium $\left(R^{2}\right)$ with lead SNP. (B, D, and F) Corresponding normal Paneth cells-numbers of risk alleles correlation. Error bars represent \pm SEM. (B) $P=2.74 \times 10^{-6} ;\left(\right.$ D) $P=5.11 \times 10^{-7} ;(\mathbf{F})$ $P=7.22 \times 10^{-7}$; all by linear regression. 


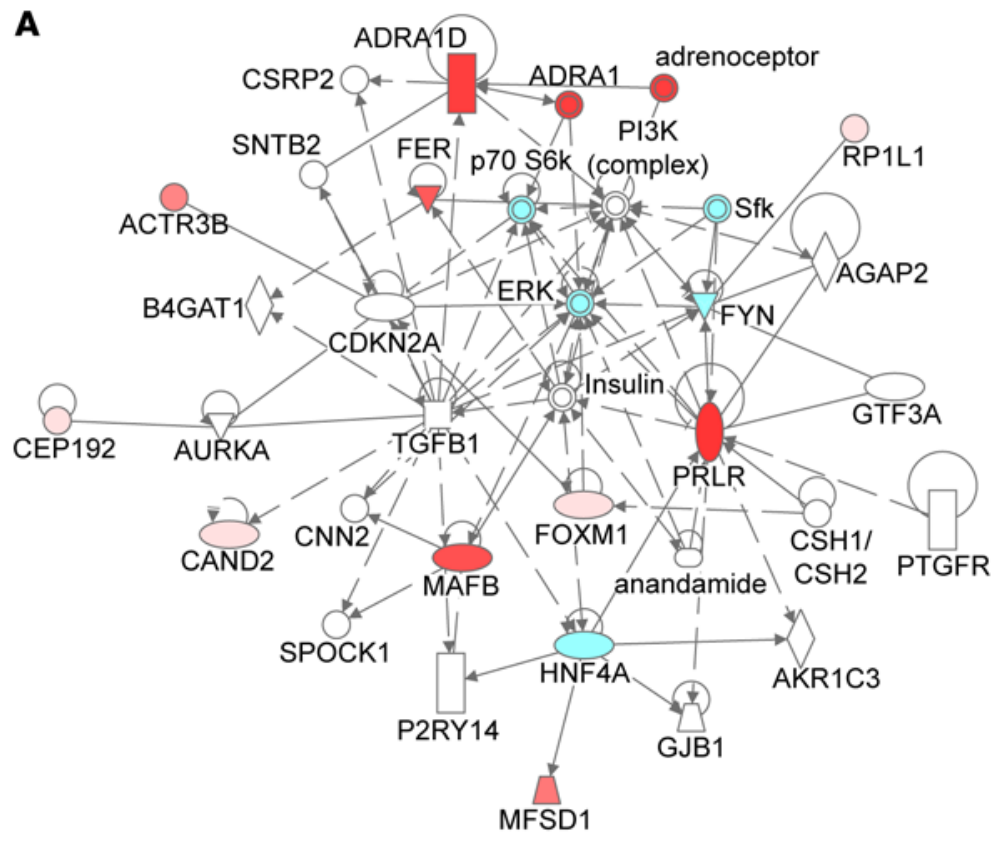

B

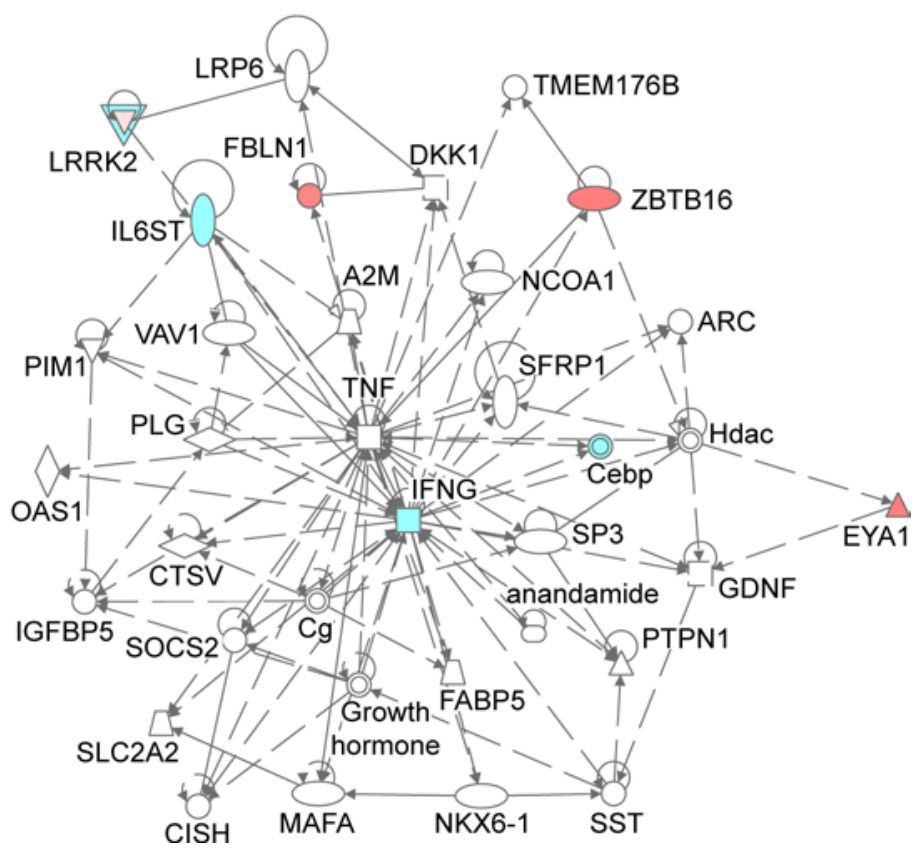

Figure 6. Ingenuity pathway analysis of the genes associated with Paneth cell defect in Japanese Crohn's disease (CD) patients. The genes include $L R R K 2$ and the candidate genes listed in Table 2 and Supplemental Table 4. The intensity of the color correlates with the $P$ values. Solid lines indicate direct interactions, and dotted lines indicate indirect interactions. Red denotes genes associated with Paneth cell defect in Japanese CD (intensity positively correlates with $P$ values). Blue denotes inflammatory bowel disease susceptibility genes. The majority of the genes associated with Paneth cell defect are involved in (A) the PI3K/mTOR pathway, which links to autophagy, whereas a minor subset of the genes are involved in (B) TNF signaling.

that had $P$ values less than or equal to $5 \times 10^{-4}$ (Supplemental Figure 4$)$. The candidate SNPs were categorized into 9 gene regions (Table 2 includes the 9 top-associated SNPs in these regions). Among the 9 candidate genes, ZBTB16, MAFB, and FER (Figure 5) were of particular interest. ZBTB16 modulates autophagy by degrading Atg14 (35). $M A F B$ is an important transcriptional factor for macrophage differentiation (36), and FER plays a role in neutrophil chemotaxis (37), both of which are involved in innate immunity and plausibly affect autophagy functions $(38,39)$. Therefore, genes involved in autophagy and innate immunity are candidates for the development of Paneth cell defect in Japanese CD. Further studies in additional cohorts are needed to confirm or refute these findings. Supplemental Table 4 shows the top functional or nonsynonymous SNPs associated with Paneth cell defect $\left(P \leq 5 \times 10^{-4}\right)$.

Pathway analysis revealed association with autophagy and inflammatory cytokines in candidate genes associated with Paneth cell defect. To determine the spectrum of pathways involved in the genes associated with Paneth cell defect in Japanese CD, we first performed gene enrichment, protein interaction, and KEGG pathway analyses to explore the biological functions of the top annotated genes corresponding to SNPs associated with abnormal Paneth cells with $P$ values less than or equal to $1 \times 10^{-3}(n=288)$ to obtain an overview of these pathways. Our results demonstrate that the gene list was enriched for specific molecular functions and biological pathways that could be related to Paneth cell phenotype. Twelve functional annotation clusters with an enrichment score of E greater than 1.4 (corresponding to unadjusted $P$ value of 0.05 ) were identified for this gene set (Supplemental Table 5). We also used the 288-gene list to generate a protein-protein interaction network (Supplemental Table 6). The functional annotation of the network is shown in Supplemental Figure 5 and the KEGG pathways $(P<$ 0.05 ) for these genes are listed in Supplemental Table 7. Many of the pathways and networks, such as cell adhesion and cytoskeletal remodeling, are known to be linked to IBD pathogenesis (4).

We next focused our analysis on the 17 identified candidate genes ( $L R R K 2$ and genes described in Table 2 and Supplemental Table 4). Ingenuity pathway analysis showed that the 17 genes identified can be connected in 2 distinct networks (Figure 6). The majority are involved in a gene network regulating PI3K and ERK signaling pathways (Figure 6A). Both of these pathways can regulate the mTOR pathway, a known upstream regulator of autophagy $(40,41)$. Moreover, in previous studies, mTOR signaling has been linked to stem cell health and Paneth cell differentiation (42-44). A second subset of genes were linked to the TNF- $\alpha$ signaling 
Table 2. Candidate SNPs and genes associated with Paneth cell defect in Japanese Crohn's disease by GWAS $\left(P \leq 5 \times 10^{-6}\right)$

\begin{tabular}{lccccccc}
\hline dbSNP & Chr & Position $^{A}$ (bp) & Allele1/2 & P value & Beta & Gene & Location \\
rs12481514 & 20 & 4225920 & A/G & $2.52 \times 10^{-7}$ & 4.469 & ADRA1D & intronic \\
rs723080 & 20 & 39357465 & C/T & $5.11 \times 10^{-7}$ & -4.754 & MAFB & upstream \\
rs147629807 & 5 & 107889038 & $-/ T$ & $7.22 \times 10^{-7}$ & -8.139 & FER & upstream \\
rs17318450 & 5 & 35482314 & A/G & $1.62 \times 10^{-6}$ & -5.826 & PRLR & upstream \\
rs12494894 & 3 & 158596415 & A/G & $2.25 \times 10^{-6}$ & -6.381 & MFSD1 & downstream \\
rs629922 & 11 & 114064825 & C/T & $2.74 \times 10^{-6}$ & 4.142 & ZBTB16 & intronic \\
rs72622838 & 8 & 72100325 & A/G & $3.04 \times 10^{-6}$ & -4.181 & EYA1 & downstream \\
rs11978753 & 7 & 152717110 & G/T & $3.65 \times 10^{-6}$ & -5.941 & ACTR3B & downstream \\
rs2238823 & 22 & 45936320 & A/G & $3.80 \times 10^{-6}$ & -5.626 & FBLN1 & intronic
\end{tabular}

${ }^{A}$ Positions are based on the Genome Reference Consortium human build 37 (GRCh37). Chr, chromosome.

pathway (Figure 6B), consistent with the central role of TNF- $\alpha$ in IBD pathogenesis $(4,6,45)$. TNF- $\alpha$ has also been implicated in the homeostasis of Paneth cell function (46-49). Therefore, the candidate genes for Paneth cell dysfunction in Japanese CD patients potentially act through modulating autophagy and TNF- $\alpha$ signaling. We also examined the potential interactions between the 17 identified genes and known CD susceptibility genes (6). We found interactions with several IBD susceptibility genes in both networks (6). For example, IBD susceptibility genes FYN, HCK, HNF4A, MAPK1, and RPS6KB1 were involved in the PI3K network, which included genes MAFB, ADRA1D, PRLR, ACTR3B, CEP192, CAND2, FOXM1, MFSD1, and RP1L1 that were identified in this study. Likewise, IBD susceptibility genes IFNG, IL6ST, LRRK2, and CEBPB, as well as genes identified in this study $L R R K 2, Z B T B 16, F B L N 1$, and $E Y A 1$, were involved in the TNF- $\alpha$ network. Therefore, the genes identified in this study potentially act in concert with known IBD susceptibility genes in autophagy and TNF- $\alpha$ signaling networks in association with Paneth cell defect.

\section{Discussion}

We have previously shown that Paneth cell phenotypes are associated with CD susceptibility alleles, pathological hallmarks, unique transcriptomic and mucosal microbiome changes, and outcome in patients undergoing surgery in North American CD $(19,20)$. However, it was unclear whether similar associations would be present in CD patients from different genetic/ethnic backgrounds, particularly as the incidence of $\mathrm{CD}$ in other ethnic groups has continued to rise $(50,51)$. In the current study, we first set out to determine the prevalence, and the genetic and clinical associations, of Paneth cell defect in Japanese CD, a population who possess a distinct spectrum of susceptibility genes compared with European ancestry CD. We demonstrated that not only was Paneth cell defect prevalent in the Japanese CD, clinically it also correlated with prognosis in Japanese CD patients after surgery. Surprisingly, there were dichotomous effects of ATG16L1 and $L R R K 2$ between the 2 cohorts. We also identified several associated SNPs in genes linked to autophagy and TNF- $\alpha$ signaling as candidates for Paneth cell defect in the Japanese CD.

While ATG16L1 T300A was associated with defective Paneth cells in both mouse models and North American adult CD patient cohorts $(18,52,53)$, this genetic association was not observed in Japanese CD. In contrast, we found that LRRK2 M2397T, a susceptibility allele for European ancestry but not for Japanese or Korean CD $(3,23,25,33,54)$, was associated with Paneth cell defect in Japanese, but not North American CD. There are several important considerations when interpreting these findings. First, given the different allele frequencies of ATG16L1 T300A between European and Asian CD cohorts (5), it is possible that a larger sample size is required to achieve sufficient power to detect an association between $A T G 16 L 1$ T300A with Paneth cell defect in Japanese CD. Second, while LRRK2 M2397T is not a susceptibility allele for Japanese CD, it could also represent a power issue in the Japanese populations. In addition, it is possible that LRRK2 M2397T, a missense variant that could result in functional defect of LRRK2, may be associated with other aspects of CD manifestations. A recent study in leprosy (a granulomatous disease) showed that patients harboring the same LRRK2 M2397T allele suffer from excessive proinflammatory responses (55). Interestingly, overlapping susceptibility loci between $\mathrm{CD}$ and mycobacterial infection (particularly leprosy) is established (3). A longitudinal study would provide additional insight into the clinical relevance of $L R R K 2$ M2397T in Japanese CD. Additional advanced sequencing technologies such as fine mapping and deep 
sequencing of the $L R R K 2$ gene and expression quantitative trait loci analysis will be important to validate the association of the M2397T SNP with Paneth cell defect, as has been shown in other studies $(22,29)$.

Using mouse models, we previously showed that Paneth cell defect is the result of gene and environment interactions, suggesting that proper environmental triggers or genetic context may be required to illicit Paneth cell defect in CD patients. The sharp contrast of divergent correlations of ATG16L1 T300A and LRRK2 M2397T with Paneth cell defects in North American and Japanese CD cohorts suggests that the environmental factors that the North American and Japanese CD patients harboring these variants encountered could be distinct. Furthermore, based on our current understanding of the autophagy machinery, in complex biologic processes such as Paneth cell function and intestinal homeostasis, the functions of these autophagy-associated genes may need to be orchestrated in distinct fashions depending on the environmental insults unique to these populations (56). One important direction is to use Paneth cell phenotype analysis as a platform to identify potential environmental factors or additional genetic factors that interact with specific host mutants in triggering Paneth cell defect in $\mathrm{CD}$ patients and mouse models with LRRK2 deficiency. In addition, environmental and genetic factors that could exert a protective effect of Paneth cell defect should be investigated through large-scale studies. Also of particular interest is to compare the Paneth cell phenotype in first- and second-generation Asian immigrants to North America (and vice versa), an approach that has yielded insight into disease pathogenesis in epidemiological studies (57-60). Based on our finding, we predict that while the genetic landscape of the second-generation immigrants will be similar to their parents, the genetic correlation to Paneth cell defects will trend more toward European ancestry North American CD. In addition, conducting genetic-Paneth cell defect analysis with focused ethnic groups (e.g., Ashkenazi Jewish) $(61,62)$ and with cross-ethnic groups (5) may provide additional insight into potential gene-gene interactions in triggering Paneth cell defect.

Among the candidate genes shown to be associated with Paneth cell defects by hypothesis-free association, $Z B T B 16, M A F B$, and FER have been shown to be linked to autophagy and components of the innate immune response that could be modulated with autophagy. ZBTB has been shown to regulate autophagy by mediating the proteasomal degradation of Atg14L (35). ZBTB16 is also involved in type 2 innate lymphoid cell function (63), NKT cell differentiation (64), and regulation of inflammatory signaling (65). MAFB is associated with macrophage differentiation $(36,66)$. FER plays a role in leukocyte recruitment and intestinal barrier function in response to bacterial lipopolysaccharide recognition (67). Both macrophage differentiation and leukocyte recruitment are important elements of innate immunity and involves autophagy (38, 39). Thus, the observation that genes involved directly and indirectly in autophagy were candidates for Paneth cell defect supports the notion that autophagy is a central pathway that controls the intestinal homeostasis (68).

We and others have found that molecular changes in processes such as autophagy and TNF signaling converge to affect Paneth cell function in CD patients and mouse models of intestinal inflammation (19, $45,52,69,70)$. This in turn correlates with the development of dysbiosis and inflammation (20). Together, these studies suggest that, at least in a subset of CD patients, Paneth cells are the origin of intestinal inflammation (10). Future studies that examine the transcriptomic and signaling pathways of Paneth cells from patients with different genetic backgrounds will provide additional insight.

Importantly, although the SNPs for Paneth cell defect identified in this study were largely distinct from the known SNPs for European ancestry CD, Paneth cell phenotype still correlated with prognosis in Japanese CD patients undergoing surgery. These results indicate that Paneth cell phenotype, as an integrated readout for combinatorial effect of host genetics and environmental factors, could potentially be applied as a universal prognostic biomarker for $\mathrm{CD}$ patients of different ethnicity/genetic backgrounds undergoing resection. Of note, a recent study has highlighted that the genes associated with CD susceptibility and prognosis are distinct (71). Therefore, development of a potential gene score that predicts postoperative recurrence may be complementary to using Paneth cell phenotype as biomarker. In addition, while we did not observe the correlation between Paneth cell phenotype and the presence of granuloma, we did observe a trend between diminished Paneth cells and granuloma, a finding that was significant in our previous study in North American CD (19). Therefore, future studies with a larger sample size may provide more insight.

In summary, we demonstrated that Paneth cell defect was prevalent in Japanese CD, was associated with different spectrum of genes compared with North American CD, and was predictive of prognosis. The genes involved in Paneth cell defect in Japanese CD mainly affect autophagy and TNF signaling pathways. Paneth cell phenotype can be applied as a universal clinical and biological relevant biomarker for CD patients with diverse genetic backgrounds. 


\section{Methods}

Subjects. A total of 110 Japanese patients with CD were included in this study. All patients were diagnosed with CD and underwent ileal resection at Tohoku University Hospital (Sendai, Japan) between 2003 and 2014. The self-reported ethnicity of all patients was Japanese. The diagnosis of CD was made based on clinical symptoms and endoscopic, radiographic, and histological findings according to conventional criteria proposed by the Japanese Ministry of Health, Labour and Welfare (72). Demographics and clinical parameters (including clinical phenotype by Montreal classification, medication received prior to and after surgery, time to disease recurrence after resection) were extracted from the medical records blinded to Paneth cell phenotypes.

In addition, a total of $164 \mathrm{CD}$ patients who underwent ileal resection were recruited at Washington University School of Medicine between 2005 and 2013, including a subset of patients $(n=50)$ that were previously reported (19).

Paneth cell phenotype analysis. Ileal resection samples previously collected as part of standard diagnostic procedures and stored in Pathology Department archives at Tohoku University and Washington University were used for Paneth cell phenotype determination. The specimens were processed as formalin-fixed, paraffin-embedded (FFPE) tissue blocks stored at $24^{\circ} \mathrm{C}$ as per routine surgical pathology practice. To be included in the study, the proximal margin tissue resection samples must have contained at least 50 welloriented intestinal crypts (73) as determined by a pathologist (T.C.L.). Paneth cell phenotype was determined by defensin-5 immunofluorescence, using defensin-5 antibody (clone 8C8; dilution 1:2,000; Novus Biologicals, catalog NB110) followed by donkey anti-mouse Alexa 488 antibody (dilution 1: 500; Thermo Fisher Scientific, catalog A-21202) as previously described (20). For a subset of cases, costaining with lysozyme immunofluorescence was performed using lysozyme antibody (clone C-19; dilution 1:100; Santa Cruz Biotechnology, catalog sc-27958) followed by donkey anti-goat Alexa 594 antibody (dilution 1: 500; Thermo Fisher Scientific, catalog A-11058). Each Paneth cell was classified as normal or abnormal based on the morphology of the cytoplasmic granules $(19,20,73)$. For prognostic correlation, the overall Paneth cell phenotype for each patient was then defined as following: type I Paneth cell phenotype was defined as greater than or equal to $20 \%$ abnormal Paneth cells, whereas type II Paneth cell phenotype was defined as less than 20\% abnormal Paneth cells $(19,20,73)$. The cutoff of $20 \%$ was based on the analysis of 106 non-IBD cases, which showed that the mean percentage of normal Paneth cells was 80.63 ( \pm 1.17 SEM). The cutoff was used in our previous studies involving North American CD cohorts $(19,20,73)$. Paneth cell phenotype analysis was performed by a pathologist (T.C.L.) who was blinded to the identification and clinical phenotype of the cases.

Definitions of clinical remission and clinical relapse. Time from index ileal resection surgery of $\mathrm{CD}$ to relapse was calculated. Endoscopy and/or computed tomography were performed when the patients had symptoms indicating recurrence (i.e., abdominal pain, worsening of diarrhea, fever, body weight loss, nausea, and appetite loss), or at least once per year if the patients had no symptoms.

Definitions of recurrence were made by endoscopy and/or radiology. Endoscopic recurrence was defined as postoperative Rutgeerts score of $i 2$ or more. Radiologic recurrence was defined by computed tomography showing bowel wall thickening (defined as a thickness $>5 \mathrm{~mm}$ ) or enhancement, fistulas, intra-abdominal abscess, and bowel obstruction. All computed tomography scans were reviewed by 2 radiologists. Patients who received immunomodulators (thiopurines), biologics (infliximab or adalimumab), or elemental diet (more than $600 \mathrm{kcal} /$ day) (74) after resection were considered to have received postoperative prophylaxis.

Genotyping. For Japanese CD patients, genomic DNA was obtained from peripheral blood leukocytes by standard phenol-chloroform extraction and precipitation or by utilizing an NA1000 Automated Nucleic Acid Extraction Machine (Kurabo) or PAXgene DNA Kit (BD). The genome-wide SNP genotypes of the Japanese CD patients were determined by the Japonica array, a SNP array designed specifically for the Japanese population (75). The array contains 659,253 SNPs, including tag SNPs for imputation, as well as SNPs related to phenotypes from previously reported GWAS and pharmacogenomics studies. As a part of quality control (QC) measures, SNPs with call rate less than $95 \%$ and samples with genotyping rates less than $95 \%$ were excluded from further analysis. Following SNP and sample QC, genotype data of 643,496 SNPs from 98 CD cases were available for further analysis. For North American CD patients, genomic DNA was extracted from whole blood $(n=50)$ as described above or from FFPE tissue using a QIAamp DNA FFPE Tissue kit (QIAGEN) $(n=114)$. For cases where whole blood was used for genomic DNA extraction, genotyping was performed with Immunochip as previously 
described (19). For cases where FFPE tissue was used for genomic DNA extraction, genotyping was performed with Taqman SNP genotyping assays (Thermo Fisher Scientific).

Imputation. Untyped genotypes were imputed in the GWAS samples using IMPUTE2 (version 2.3.2) and 1,070 healthy individuals from Japan ( $1 \mathrm{KJPN}$ panel, which contains $>20$ million SNPs) (76) as a reference dataset. SNPs with low imputation quality (with a posterior probability score of $<0.90$ ), minor allele frequency less than 0.10 and Hardy-Weinberg equilibrium $P$ value less than $1 \times 10^{-5}$ were excluded. After exclusion, genotype data of 4,198,245 SNPs from 98 Japanese CD cases were used for further analysis.

Pathway and network analysis. Gene enrichment and network analysis was performed on the top 288 annotated genes corresponding to the SNPs with $P$ less than or equal to $1 \times 10^{-3}$ (from linear regression) using online bioinformatics tools DAVID (https://david.ncifcrf.gov/tools.jsp) and STRING (http://www. string-db.org/). Functional annotation clusters corresponding to the gene list were identified using DAVID. Protein-protein interaction (PPI) network for the 288 genes was extracted using STRING. An annotation network was constructed by combining function-based information with PPI network information and visualized using Cytoscape (www.cytoscape.org). Corresponding pathways $(P<0.05)$ were annotated using the enrichment analysis tool in STRING. Core pathway analysis of the 17 candidate genes was performed using Ingenuity Pathway Analysis software (QIAGEN).

Statistics. The percentages of normal Paneth cells between North American CD and Japanese CD were compared by unpaired $t$ test, and the prevalence of type I Paneth cell phenotype between the 2 groups was compared using Fisher's exact test. For genotype-Paneth cell defect correlation, we first adopted a hypothesis-driven approach with selected SNPs (Figure 1). These SNPs include: (a) 45 SNPs shown to have CD-specific susceptibility from an ImmunoChip study examining European ancestry CD (3); (b) an additional 38 SNPs reported in European and East Asian ancestry CD (5); (c) 14 SNPs associated with East Asian (Japanese and/or Korean) CD through GWAS analyses (21, 23, 28, 77) and deep-resequencing analysis (22); and (d) 2 functional CD-associated variants that were either reported to be associated with Paneth cell defect in our previous studies $(18,19)$, or whose genes involved had been implicated to be associated with Paneth cell biology in preclinical studies $(17,29)$. By excluding duplicates, a total of 73 SNPs were compiled as candidates. Among the 73 SNPs, 14 SNPs were very rare (minor allele frequency $<1.0 \%$ ) or nonpolymorphic (i.e., NOD2 variants) in East Asian, and 3 SNPs were not constructed/included on the Japonica array. After excluding these 17 SNPs, a final 56 SNPs were selected for the hypothesis-driven approach. After Bonferroni correction, SNPs with $P$ values less than $8.93 \times 10^{-4}$ were considered significant. For hypothesis-driven analyses, we correlated the genotypes with the percentage of normal Paneth cells by linear regression.

For the subsequent unbiased, GWAS analysis (Figure 1), linear regression was performed using PLINK v1.07 software (78). SNPs with $P$ values less than $5 \times 10^{-8}$ were considered genome-wide significant. SNPs with $P$ values less than $5 \times 10^{-6}$ and nonsynonymous SNPs with $P$ values less than $5 \times 10^{-4}$ were considered candidates with nominal significance.

SNPs located within $100 \mathrm{kbp}$ were considered to be in 1 region. Genes within $200 \mathrm{kbp}$ of candidate SNPs were investigated. Correlation with time to disease recurrence after surgery was performed using the log-rank test and Cox-proportional hazards model. Manhattan plots were generated using the R package qqman, regional association plots were generated using LocusZoom application (79). All statistical analyses, except genome-wide linear regression, were performed using JMP 11 (SAS Institute Inc.), GraphPad Prism (version 6.5), or R software (version 3.1.3).

Power calculation. Using the software Quanto, we calculated the power of the current study to detect genetic variants associated with the Paneth cell phenotype, and demonstrated that in the Japanese cohort, we have a power of $80.66 \%$ to detect a variant with variance contribution of 0.07 at 0.05 significance threshold for the hypothesis-driven approach. In the Washington University cohort, we have a power of $82.65 \%$ to detect a variant with variance contribution of 0.05 with a significance threshold of 0.05 . At genome-wide significance threshold $\left(5 \times 10^{-8}\right)$, the Japanese cohort has a power of $56.91 \%$ to detect a variant with variance contribution of 0.247 .

Study approval. The study was approved by the Ethics Committee of Tohoku University Graduate School of Medicine under protocol number 2013-1-539 and the IRB of Washington University School of Medicine under protocol number 201209047. Subjects provided informed consent prior to their participation in the study.

Data depository. The accession number for the genotyping data deposition at NCBI Gene Expression Omnibus repository is GSE90102. 


\section{Author contributions}

TCL, TN, DPBM, Y. Kakuta, and TSS designed the study. TCL, TN, ZL, TH, Y. Kawai, MN, and Y. Kakuta acquired data. TN, TS, and TK recruited patients. TCL, TN, ZL, KLV, TH, DL, DPPM, Y. Kakuta, and TSS analyzed data. TCL, TN, DPBM, Y. Kakuta, and TSS drafted the manuscript. TN, KE, and Y. Kinouchi provided samples.

\section{Acknowledgments}

The study was supported by the Helmsley Charitable Trust (D.P.B.M., T.S.S.), Doris Duke Charitable Foundation grant 2014103 (T.C.L.), Crohn's and Colitis Foundation grant 274415, NIH grants 1R56DK095820 (D.P.B.M., T.S.S.) and K01DK109081 (K.L.V.), NIH/National Center for Advancing Translational Sciences (NCATS) grant UL1 TR000448 (T.C.L.), and Japan Society for the Promotion of Science KAKENHI grants JP15H04805 and JP15K15284 (Y.K.). Computational resources for genotype imputation were provided by the Tohoku Medical Megabank Organization supercomputer system.

Address correspondence to: Thaddeus S. Stappenbeck or Ta-Chiang Liu, Department of Pathology and Immunology, Washington University School of Medicine, 660 South Euclid Avenue, CB 8118, St. Louis, Missouri 63110, USA. Phone: 314.362.4214; E-mail: stappenb@wustl.edu (T.S. Stappenbeck). Phone: 314.747.0343; E-mail: tliu27@wustl.edu (T.C. Liu). Or to: Yoichi Kakuta, Division of Gastroenterology, Tohoku University Graduate School of Medicine, 1-1 Seiryo, Aoba, Sendai, 980-8574, Japan. Phone: 81.22.717.7171; E-mail: ykakuta@med.tohoku.ac.jp.

1. Baumgart DC, Sandborn WJ. Crohn's disease. Lancet. 2012;380(9853):1590-1605.

2. Ordás I, Eckmann L, Talamini M, Baumgart DC, Sandborn WJ. Ulcerative colitis. Lancet. 2012;380(9853):1606-1619.

3. Jostins L, et al. Host-microbe interactions have shaped the genetic architecture of inflammatory bowel disease. Nature. 2012;491(7422):119-124.

4. Khor B, Gardet A, Xavier RJ. Genetics and pathogenesis of inflammatory bowel disease. Nature. 2011;474(7351):307-317

5. Liu JZ, et al. Association analyses identify 38 susceptibility loci for inflammatory bowel disease and highlight shared genetic risk across populations. Nat Genet. 2015;47(9):979-986.

6. McGovern DP, Kugathasan S, Cho JH. Genetics of inflammatory bowel diseases. Gastroenterology. 2015;149(5):1163-1176.e2.

7. Liu TC, Stappenbeck TS. Genetics and pathogenesis of inflammatory bowel disease. Annu Rev Pathol. 2016;11:127-148.

8. Lewis JD. The utility of biomarkers in the diagnosis and therapy of inflammatory bowel disease. Gastroenterology. 2011;140(6):1817-1826.e2.

9. Bevins CL, Salzman NH. Paneth cells, antimicrobial peptides and maintenance of intestinal homeostasis. Nat Rev Microbiol. 2011;9(5):356-368.

10. Adolph TE, et al. Paneth cells as a site of origin for intestinal inflammation. Nature. 2013;503(7475):272-276.

11. Clevers HC, Bevins CL. Paneth cells: maestros of the small intestinal crypts. Annu Rev Physiol. 2013;75:289-311

12. Salzman NH, et al. Enteric defensins are essential regulators of intestinal microbial ecology. Nat Immunol. 2010;11(1):76-83.

13. Salzman NH, Chou MM, de Jong H, Liu L, Porter EM, Paterson Y. Enteric salmonella infection inhibits Paneth cell antimicrobial peptide expression. Infect Immun. 2003;71(3):1109-1115.

14. Wehkamp J, et al. Reduced Paneth cell alpha-defensins in ileal Crohn's disease. Proc Natl Acad Sci USA. 2005;102(50):18129-18134.

15. Bevins CL, Stange EF, Wehkamp J. Decreased Paneth cell defensin expression in ileal Crohn's disease is independent of inflammation, but linked to the NOD2 1007fs genotype. Gut. 2009;58(6):882-3; discussion 883.

16. Ouellette AJ. Paneth cells and innate mucosal immunity. Curr Opin Gastroenterol. 2010;26(6):547-553.

17. Kaser A, et al. XBP1 links ER stress to intestinal inflammation and confers genetic risk for human inflammatory bowel disease. Cell. 2008;134(5):743-756.

18. Cadwell K, et al. A key role for autophagy and the autophagy gene Atg1611 in mouse and human intestinal Paneth cells. Nature. 2008;456(7219):259-263.

19. VanDussen KL, et al. Genetic variants synthesize to produce paneth cell phenotypes that define subtypes of Crohn's disease. Gastroenterology. 2014;146(1):200-209.

20. Liu TC, et al. Paneth cell defects in Crohn's disease patients promote dysbiosis. JCI Insight. 2016;1(8):e86907.

21. Yamazaki K, et al. A genome-wide association study identifies 2 susceptibility Loci for Crohn's disease in a Japanese population. Gastroenterology. 2013;144(4):781-788.

22. Hong SN, et al. Deep resequencing of 131 Crohn's disease associated genes in pooled DNA confirmed three reported variants and identified eight novel variants. Gut. 2016;65(5):788-796.

23. Yang SK, et al. Genome-wide association study of Crohn's disease in Koreans revealed three new susceptibility loci and common attributes of genetic susceptibility across ethnic populations. Gut. 2014;63(1):80-87.

24. Nakagome S, et al. Population-specific susceptibility to Crohn's disease and ulcerative colitis; dominant and recessive relative risks in the Japanese population. Ann Hum Genet. 2010;74(2):126-136.

25. Hirano A, et al. Association study of 71 European Crohn's disease susceptibility loci in a Japanese population. Inflamm Bowel Dis. 2013;19(3):526-533

26. Sorrentino D. State-of-the-art medical prevention of postoperative recurrence of Crohn's disease. Nat Rev Gastroenterol Hepatol. 
2013;10(7):413-422.

27. Hampe J, et al. A genome-wide association scan of nonsynonymous SNPs identifies a susceptibility variant for Crohn disease in ATG16L1. Nat Genet. 2007;39(2):207-211.

28. Yang SK, et al. Immunochip analysis identification of 6 additional susceptibility loci for Crohn's disease in Koreans. Inflamm Bowel Dis. 2015;21(1):1-7.

29. Trabzuni D, et al. Fine-mapping, gene expression and splicing analysis of the disease associated LRRK2 locus. PLoS ONE. 2013;8(8):e70724.

30. Cadwell K, et al. Virus-plus-susceptibility gene interaction determines Crohn's disease gene Atg16L1 phenotypes in intestine Cell. 2010;141(7):1135-1145.

31. Inoue N, et al. Lack of common NOD2 variants in Japanese patients with Crohn's disease. Gastroenterology. 2002;123(1):86-91.

32. Yamazaki K, Takazoe M, Tanaka T, Kazumori T, Nakamura Y. Absence of mutation in the NOD2/CARD15 gene among 483 Japanese patients with Crohn's disease. J Hum Genet. 2002;47(9):469-472.

33. Franke A, et al. Genome-wide meta-analysis increases to 71 the number of confirmed Crohn's disease susceptibility loci. Nat Genet. 2010;42(12):1118-1125.

34. Zhang Q, et al. Commensal bacteria direct selective cargo sorting to promote symbiosis. Nat Immunol. 2015;16(9):918-926.

35. Zhang T, et al. G-protein-coupled receptors regulate autophagy by ZBTB16-mediated ubiquitination and proteasomal degradation of Atg14L. Elife. 2015;4:e06734.

36. Aziz A, Soucie E, Sarrazin S, Sieweke MH. MafB/c-Maf deficiency enables self-renewal of differentiated functional macrophages. Science. 2009;326(5954):867-871.

37. Khajah M, Andonegui G, Chan R, Craig AW, Greer PA, McCafferty DM. Fer kinase limits neutrophil chemotaxis toward end target chemoattractants. J Immunol. 2013;190(5):2208-2216.

38. Chen P, Cescon M, Bonaldo P. Autophagy-mediated regulation of macrophages and its applications for cancer. Autophagy. 2014;10(2):192-200

39. Kanayama M, Inoue M, Danzaki K, Hammer G, He YW, Shinohara ML. Autophagy enhances NFKB activity in specific tissue macrophages by sequestering A20 to boost antifungal immunity. Nat Commun. 2015;6:5779.

40. Shanware NP, Bray K, Abraham RT. The PI3K, metabolic, and autophagy networks: interactive partners in cellular health and disease. Annu Rev Pharmacol Toxicol. 2013;53:89-106.

41. Jung CH, Ro SH, Cao J, Otto NM, Kim DH. mTOR regulation of autophagy. FEBS Lett. 2010;584(7):1287-1295.

42. Zhou Y, Rychahou P, Wang Q, Weiss HL, Evers BM. TSC2/mTORC1 signaling controls Paneth and goblet cell differentiation in the intestinal epithelium. Cell Death Dis. 2015;6:e1631.

43. Igarashi M, Guarente L. mTORC1 and SIRT1 cooperate to foster expansion of gut adult stem cells during calorie restriction. Cell. 2016;166(2):436-450.

44. Yilmaz ÖH, et al. mTORC1 in the Paneth cell niche couples intestinal stem-cell function to calorie intake. Nature. 2012;486(7404):490-495.

45. Günther C, et al. Caspase-8 regulates TNF- $\alpha$-induced epithelial necroptosis and terminal ileitis. Nature. 2011;477(7364):335-339.

46. Lu W, de Leeuw E. Functional intersection of human defensin 5 with the TNF receptor pathway. FEBS Lett. 2014;588(10):1906-1912.

47. Van Hauwermeiren F, et al. TNFR1-induced lethal inflammation is mediated by goblet and Paneth cell dysfunction. Mucosal Immunol. 2015;8(4):828-840.

48. Schaubeck M, et al. Dysbiotic gut microbiota causes transmissible Crohn's disease-like ileitis independent of failure in antimicrobial defence. Gut. 2016;65(2):225-237.

49. Roulis M, et al. Host and microbiota interactions are critical for development of murine Crohn's-like ileitis. Mucosal Immunol. 2016;9(3):787-797.

50. Ooi CJ, et al. Asia Pacific Consensus Statements on Crohn's disease. Part 1: Definition, diagnosis, and epidemiology: (Asia Pacific Crohn's Disease Consensus--Part 1). J Gastroenterol Hepatol. 2016;31(1):45-55.

51. Malhotra R, Turner K, Sonnenberg A, Genta RM. High prevalence of inflammatory bowel disease in United States residents of Indian ancestry. Clin Gastroenterol Hepatol. 2015;13(4):683-689.

52. Lassen KG, et al. Atg16L1 T300A variant decreases selective autophagy resulting in altered cytokine signaling and decreased antibacterial defense. Proc Natl Acad Sci USA. 2014;111(21):7741-7746.

53. Murthy A, et al. A Crohn's disease variant in Atg1611 enhances its degradation by caspase 3. Nature. 2014;506(7489):456-462.

54. Fuyuno Y, et al. Genetic characteristics of inflammatory bowel disease in a Japanese population. $J$ Gastroenterol. 2016;51(7):672-681.

55. Fava VM, et al. A missense LRRK2 variant is a risk factor for excessive inflammatory responses in leprosy. PLoS Negl Trop Dis. 2016;10(2):e0004412.

56. Levine B, Mizushima N, Virgin HW. Autophagy in immunity and inflammation. Nature. 2011;469(7330):323-335.

57. Ko Y, Butcher R, Leong RW. Epidemiological studies of migration and environmental risk factors in the inflammatory bowel diseases. World J Gastroenterol. 2014;20(5):1238-1247.

58. Probert CS, Jayanthi V, Pinder D, Wicks AC, Mayberry JF. Epidemiological study of ulcerative proctocolitis in Indian migrants and the indigenous population of Leicestershire. Gut. 1992;33(5):687-693.

59. Carr I, Mayberry JF. The effects of migration on ulcerative colitis: a three-year prospective study among Europeans and firstand second-generation South Asians in Leicester (1991-1994). Am J Gastroenterol. 1999;94(10):2918-2922.

60. Li X, Sundquist J, Hemminki K, Sundquist K. Risk of inflammatory bowel disease in first- and second-generation immigrants in Sweden: a nationwide follow-up study. Inflamm Bowel Dis. 2011;17(8):1784-1791.

61. Chuang LS, et al. A frameshift in CSF2RB predominant among Ashkenazi Jews increases risk for Crohn's disease and reduces monocyte signaling via GM-CSF. Gastroenterology. 2016;151(4):710-723.e2.

62. Vacic V, et al. Genome-wide mapping of IBD segments in an Ashkenazi PD cohort identifies associated haplotypes. Hum Mol Genet. 2014;23(17):4693-4702. 
63. Verhoef PA, Constantinides MG, McDonald BD, Urban JF, Sperling AI, Bendelac A. Intrinsic functional defects of type 2 innate lymphoid cells impair innate allergic inflammation in promyelocytic leukemia zinc finger (PLZF)-deficient mice. $J$ Allergy Clin Immunol. 2016;137(2):591-600.e1.

64. Pobezinsky LA, et al. Let-7 microRNAs target the lineage-specific transcription factor PLZF to regulate terminal NKT cell differentiation and effector function. Nat Immunol. 2015;16(5):517-524.

65. Sadler AJ, et al. BTB-ZF transcriptional regulator PLZF modifies chromatin to restrain inflammatory signaling programs. Proc Natl Acad Sci USA. 2015;112(5):1535-1540.

66. Soucie EL, et al. Lineage-specific enhancers activate self-renewal genes in macrophages and embryonic stem cells. Science. 2016;351(6274):aad5510.

67. Qi W, Ebbert KV, Craig AW, Greer PA, McCafferty DM. Absence of Fer protein tyrosine kinase exacerbates endotoxin induced intestinal epithelial barrier dysfunction in vivo. Gut. 2005;54(8):1091-1097.

68. Baxt LA, Xavier RJ. Role of autophagy in the maintenance of intestinal homeostasis. Gastroenterology. 2015;149(3):553-562.

69. Tschurtschenthaler M, et al. Type I interferon signalling in the intestinal epithelium affects Paneth cells, microbial ecology and epithelial regeneration. Gut. 2014;63(12):1921-1931.

70. Deuring JJ, et al. Genomic ATG16L1 risk allele-restricted Paneth cell ER stress in quiescent Crohn's disease. Gut. 2014;63(7):1081-1091

71. Lee JC, et al. Genome-wide association study identifies distinct genetic contributions to prognosis and susceptibility in Crohn's disease. Nat Genet. 2017;49(2):262-268.

72. Matsui T, Hirai F, Hisabe T. Proposed diagnostic criteria for Crohn's disease. Annual reports of the research group of intractable inflammatory bowel disease subsidized by the Ministry of Health, Labour, and Welfare of Japan, Tokyo, Japan. 2011;52-54 .

73. Liu TC, Gao F, McGovern DP, Stappenbeck TS. Spatial and temporal stability of paneth cell phenotypes in Crohn's disease: implications for prognostic cellular biomarker development. Inflamm Bowel Dis. 2014;20(4):646-651.

74. Takagi S, et al. Effectiveness of an 'half elemental diet' as maintenance therapy for Crohn's disease: A randomized-controlled trial. Aliment Pharmacol Ther. 2006;24(9):1333-1340.

75. Kawai Y, et al. Japonica array: improved genotype imputation by designing a population-specific SNP array with 1070 Japanese individuals. J Hum Genet. 2015;60(10):581-587.

76. Nagasaki M, et al. Rare variant discovery by deep whole-genome sequencing of 1,070 Japanese individuals. Nat Commun $2015 ; 6: 8018$.

77. Yamazaki K, et al. Single nucleotide polymorphisms in TNFSF15 confer susceptibility to Crohn's disease. Hum Mol Genet. 2005;14(22):3499-3506

78. Purcell S, et al. PLINK: a tool set for whole-genome association and population-based linkage analyses. Am J Hum Genet. 2007;81(3):559-575

79. Pruim RJ, et al. LocusZoom: regional visualization of genome-wide association scan results. Bioinformatics. 2010;26(18):2336-2337. 